\title{
Mediastinal Epithelioid Hemangioendothelioma
}

National Cancer Institute

\section{Source}

National Cancer Institute. Mediastinal Epithelioid Hemangioendothelioma. NCI

Thesaurus. Code C146988.

An epithelioid hemangioendothelioma that arises from the mediastinum. 